\title{
Expression of $\alpha$-taxilin in hepatocellular carcinoma correlates with growth activity and malignant potential of the tumor
}

\author{
NATSUKO OHTOMO ${ }^{1}$, TOMOAKI TOMIYA ${ }^{1}$, YASUSHI TANOUE ${ }^{1}$, YUKIKO INOUE ${ }^{1}$, TAKAKO NISHIKAWA ${ }^{1}$, \\ HITOSHI IKEDA ${ }^{4}$, YASUJI SEYAMA ${ }^{2}$, NORIHIRO KOKUDO ${ }^{2}$, JUNJI SHIBAHARA ${ }^{3}$, MASASHI FUKAYAMA ${ }^{3}$, \\ KAZUHIKO KOIKE $^{1}$, HIROMICHI SHIRATAKI ${ }^{5}$ and KENJI FUJIWARA ${ }^{6}$ \\ Departments of ${ }^{1}$ Gastroenterology, ${ }^{2}$ Hepato-Biliary-Pancreatic Surgery and ${ }^{3}$ Pathology, University of Tokyo; \\ ${ }^{4}$ Clinical Laboratory, University of Tokyo Hospital, 7-3-1 Hongo, Bunkyo-ku, Tokyo; ${ }^{5}$ Molecular and Cell Biology, \\ Dokkyo Medical School, 880 Kitakobayashi, Mibu-machi, Shimotsuga-gun, Tochigi; \\ ${ }^{6}$ Yokohama Rosai Hospital, 3211 Kodukue-cho, Kohoku-ku, Yokohama, Kanagawa, Japan
}

Received July 20, 2010; Accepted September 14, 2010

DOI: 10.3892/ijo_00000793

\begin{abstract}
The membrane traffic system has been recognized to be involved in carcinogenesis and tumor progression in several types of tumors. $\alpha$-taxilin is a newly identified membrane traffic-related molecule, and its up-regulation has been reported in embryonic and malignant tissues of neural origin. In the present study, we analyzed the expression of $\alpha$-taxilin in relation to clinicopathological features of hepatocellular carcinomas (HCC) and proliferative activity of the tumor determined by proliferating cell nuclear antigen labeling index (PCNA-LI). Twenty-nine surgically resected nodules of HCC (8 well-, 11 moderately-, and 10 poorlydifferentiated) were studied. Fifteen cases showed 'strong staining', while 14 cases showed 'weak staining' for $\alpha$-taxilin. A significantly higher expression of $\alpha$-taxilin was observed in less-differentiated $(\mathrm{p}=0.005)$, and more invasive $(\mathrm{p}=0.016)$ HCCs. The 'strong staining' group showed significantly higher PCNA-LI than the 'weak staining' group (the medians of PCNA-LI were $59.4 \%$ vs. $14.4 \%, \mathrm{p}<0.0001)$. We also evaluated the expression of $\alpha$-taxilin in hepatoma cell lines (PLC/PRF/5, Hep G2 and HuH-6) in association with cell proliferation. The expression levels of $\alpha$-taxilin protein were correlated with their growth rates. In conclusion, the expression of the $\alpha$-taxilin protein was related with an increased proliferative activity and a less-differentiated histological grade of HCC. $\alpha$-taxilin may be involved in cell proliferation of $\mathrm{HCC}$, and its expression can be a marker of malignant potential of HCC.
\end{abstract}

Correspondence to: Dr Tomoaki Tomiya, Department of Gastroenterology, University of Tokyo, 7-3-1 Hongo, Bunkyo-ku, Tokyo, Japan

E-mail: tomiya-1im@h.u-tokyo.ac.jp

Key words: hepatocellular carcinoma, taxilin, vesicular transport proteins

\section{Introduction}

The molecular mechanisms of carcinogenesis and tumor progression have been extensively investigated to find novel targets for anti-tumor therapy as well as useful predictors of tumor growth and biological aggressiveness. Involvement of intracellular signaling pathways, cell cycle regulators, growth factors and angiogenic factors has been shown in a variety of carcinomas $(1,2)$. Furthermore, recent studies have revealed that membrane traffic-related molecules play a role in the processes of carcinogenesis and tumor progression in several types of tumors (3-5).

Membrane traffic is a fundamental intracellular transport system in eukaryotic cells (6). Small transport vesicles bud from membranes of a donor compartment, and subsequently fuse with membranes of a target compartment. Cargo molecules in the vesicles as well as biomembranes are dynamically exchanged between organelles with temporal and spatial selectivity. These processes are essential for multiple cellular functions such as endo- and exocytosis, maintenance of organelle homeostasis and cell growth, division, and motility. Soluble $N$-ethylmaleimide-sensitive factor attachment protein receptors (SNAREs), located on vesicles and the target membranes, are the central coordinators of membrane traffic (7), and syntaxin family proteins are their main components.

Recently, taxilin was identified as a novel binding partner of syntaxins (8). $\alpha$-taxilin, one of the isoforms, was proposed to be involved in $\mathrm{Ca}^{++}$-dependent exocytosis in neuroendocrine cells, although its actual function in vivo is not yet known. Over-expression of $\alpha$-taxilin mRNA has been reported in human glioblastoma compared to normal tissues of the central nervous system (CNS) (9). Prominent up-regulation of $\alpha$-taxilin protein has also been reported in proliferating neural stem cells during embryonic development in rats followed by a rapid decrease of the expression level as development proceeds (10). These findings imply that $\alpha$-taxilin is related to cell proliferation of mesenchymal cells, especially in the CNS. However, significance of the expression of $\alpha$-taxilin protein has not yet been studied in tissues or malignancies of epithelial origin. 
In the present study, we analyzed the expression of $\alpha$-taxilin in hepatocellular carcinomas (HCC), which is one of the most common malignancies of epithelial origin worldwide and is known to have wide varieties of differentiation and growth activity. We found a correlation of $\alpha$-taxilin expression with proliferative activity as well as with malignant potential of HCC.

\section{Patients and methods}

Patients. Twenty-nine patients of HCC were studied who underwent curative or non-curative hepatectomy at the Hepato-Biliary-Pancreatic and Transplantation Division between May 2003 and August 2008. No patients received preoperative treatments for HCC. Diagnosis of HCC was confirmed by histology based on sections stained with hematoxylin and eosin. The degree of tumor differentiation and other pathological features were assessed according to the Liver Cancer Study Group of Japan (11), and reviewed by a pathologist who was not informed of the results of $\alpha$ taxilin expression. When there were multiple nodules of HCC in the resected tissues, the largest one was considered to be representative. The clinical and pathological features of the study population are shown in Table I. Informed consent was obtained from each participant. This study protocol was approved by the institutional review board of the University of Tokyo.

Immunohistochemistry of $\alpha$-taxilin. The polyclonal anti-human $\alpha$-taxilin antibody raised in a rabbit was used for immunohistochemistry, as previously described (12).

The formalin-fixed, paraffin-embedded liver specimens containing HCC nodules were sliced into $3 \mu \mathrm{m}$-thick sections, immersed in xylene and graded alcohols, and washed in $10 \mathrm{mM}$ PBS. The sections were heated using a microwave processor MI-77 (Azumaya, Tokyo, Japan) in $10 \mathrm{mM}$ citrate buffer $(\mathrm{pH} 6.0)$ at $95^{\circ} \mathrm{C}$ for $30 \mathrm{~min}$. The sections were treated with $0.3 \% \mathrm{v} / \mathrm{v} \mathrm{H}_{2} \mathrm{O}_{2}$ in methanol at room temperature for $30 \mathrm{~min}$, blocking solution $(10 \% \mathrm{v} / \mathrm{v}$ normal goat serum and $1 \% \mathrm{w} / \mathrm{v}$ BSA in $10 \mathrm{mM}$ PBS) at room temperature for $1 \mathrm{~h}$, and then incubated with a primary antibody diluted in the blocking solution overnight at $4^{\circ} \mathrm{C}$.

A standard avidin-biotin-peroxidase complex (ABC) technique with Vectastain ${ }^{\circledR}$ ABC elite kit (Vector Laboratories, Burlingame, CA, USA) was applied. Briefly, a biotinylated antibody against rabbit IgG diluted in the blocking solution and $\mathrm{ABC}$ solution were applied at room temperature for 25 and $30 \mathrm{~min}$, respectively. Staining was visualized with 3,3'diaminobenzidine working solution (Vector Laboratories) with nuclear counterstaining in Mayer's hematoxylin (Wako Pure Chemical Industries Ltd., Osaka, Japan).

The sections were studied with a light microscope Eclipse $80 \mathrm{i}$ (Nikon, Tokyo, Japan) at magnifications x40, x100 and x200 with a digital camera DXM1200F (Nikon). Intensity of $\alpha$-taxilin staining was evaluated independently by three of the authors. The intensity of staining in HCC tissues was classified into two categories: 'strong staining' where almost all the cancerous cells were stained (Fig. 1A), and 'weak staining' where no stained cells or few weakly stained cells were observed in cancerous tissues (Fig. 1B).
Immunohistochemistry of proliferating cell nuclear antigen (PCNA) in HCC. Immunohistochemistry of PCNA was performed on the serial section of the specimens as mentioned above except for blocking solution (10\% v/v normal horse serum and $1 \% \mathrm{w} / \mathrm{v}$ BSA in $10 \mathrm{mM}$ PBS), the primary antibody (mouse monoclonal anti-PCNA antibody (clone PC10; dilution 1:200; Dako, Glostrup, Denmark)), the secondary antibody (a biotinylated anti-mouse $\mathrm{IgG}$ ) and the duration of microwave heating (10 $\mathrm{min})$.

PCNA labeling index (PCNA-LI) was determined by random evaluation of at least $1,000 \mathrm{HCC}$ cell nuclei at magnification $x 100$. All of the stained nuclei were regarded as positive. PCNA-LI was expressed as the percentage of positive nuclei $(13,14)$.

Preparation of frozen tissue samples of HCC for Western blotting. Western blotting was performed on fresh frozen tissue samples from one representative case showing 'strong staining' of $\alpha$-taxilin. The samples obtained at hepatectomy from the HCC nodule and adjacent non-cancerous liver tissue were snap-frozen in liquid nitrogen, and stored at $-80^{\circ} \mathrm{C}$. They were homogenized in ice-cold buffer containing $20 \mathrm{mM}$ Tris/HCl (pH 7.5), $150 \mathrm{mM} \mathrm{NaCl}, 1 \mathrm{mM}$ EDTA, Protease inhibitor cocktail Complete ${ }^{\circledR}$ (Roche Diagnostics, Basel, Switzerland), $1 \mathrm{mM}$ DTT, and 2\% w/v Triton X-100, incubated for $1 \mathrm{~h}$ with intermittent vortexing.

Preparation of lysates of hepatoma cell lines for Western blotting. The human hepatoma cell lines, Hep G2 (cell no. JCRB1054), PLC/PRF/5 (JCRB0406) and HuH-6 Clone 5 (JCRB0401) were purchased from Health Science Research Resources Bank (Osaka, Japan) in November 2008. The cell lines were authorized by Multiplex PCR method using short tandem repeat by the cell bank.

The cells were plated on plastic culture dishes (BD, Franklin Lakes, NJ, USA) at a density of 3,000 cells $/ \mathrm{cm}^{2}$ in DMEM with $10 \%$ v/v FCS and grown to $50-70 \%$ confluency. The cells were lysed in ice-cold buffer containing $50 \mathrm{mM}$ Tris/ $\mathrm{HCl}$ (pH 8.0), $120 \mathrm{mM} \mathrm{NaCl}, 20 \mathrm{mM} \mathrm{NaF}, 1 \mathrm{mM}$ EDTA, $6 \mathrm{mM}$ EGTA, $0.5 \mathrm{mM}$ DTT, protease inhibitor cocktail, and $1 \% \mathrm{v} / \mathrm{v}$ NP-40, incubated for $10 \mathrm{~min}$ with intermittent vortexing.

Western blotting for a-taxilin. Protein concentrations of the tissue homogenates and the cell lysates were determined by the Lowry's method with DC ${ }^{\mathrm{TM}}$ protein assay kit (Bio-Rad Laboratories, Hercules, CA, USA). The samples were adjusted to the same protein concentration, boiled at $95^{\circ} \mathrm{C}$ for $5 \mathrm{~min}$ with a half volume of SDS buffer (186 mM Tris/HCl ( $\mathrm{pH} 6.7)$, 9\% SDS, $15 \%$ glycerol, 6\% 2-mercaptoethanol, bromophenol blue), separated by SDS-PAGE on a $10 \%$ polyacrylamide gel, and transferred to PVDF membranes (GE Healthcare, Buckinghamshire, UK). The membranes were treated with blocking buffer (10 mM PBS containing 5\% w/v skim milk and $0.1 \% \mathrm{w} / \mathrm{v}$ Tween-20) for $1 \mathrm{~h}$ at room temperature, and incubated with $0.1 \mu \mathrm{g} / \mathrm{ml}$ of anti-human $\alpha$-taxilin antibody in the blocking buffer overnight at $4^{\circ} \mathrm{C}$, and subsequently with HRP-conjugated anti-rabbit IgG (GE Healthcare) in the blocking buffer (dilution 1:2,000) for $45 \mathrm{~min}$ at room temperature. The chemiluminescent signals were visualized 
Table I. $\alpha$-taxilin expression in HCC and clinical and pathological features.

\begin{tabular}{|c|c|c|c|c|}
\hline & \multirow[b]{2}{*}{ All } & \multicolumn{2}{|c|}{ Intensity of $\alpha$-taxilin expression } & \multirow[b]{2}{*}{ P-value } \\
\hline & & Weak $(\%)$ & Strong $(\%)$ & \\
\hline Age $^{a}$ & $68.0(45-76)$ & $68.0(51-76)$ & $68.0(45-76)$ & \\
\hline$<68$ & 14 & $7(50)$ & $7(50)$ & 0.860 \\
\hline$\geq 68$ & 15 & $7(47)$ & $8(53)$ & \\
\hline \multicolumn{5}{|l|}{ Gender } \\
\hline Male & 24 & $10(42)$ & $14(58)$ & 0.125 \\
\hline Female & 5 & $4(80)$ & 1 (20) & \\
\hline \multicolumn{5}{|l|}{ Background liver $^{b}$} \\
\hline Liver cirrhosis & 17 & $10(59)$ & $7(41)$ & 0.395 \\
\hline Chronic hepatitis & 10 & $4(40)$ & $6(60)$ & \\
\hline Normal liver & 1 & $0 \quad(0)$ & $1(100)$ & \\
\hline \multicolumn{5}{|l|}{ Number of tumors } \\
\hline Solitary & 20 & $10(50)$ & $10(50)$ & 0.786 \\
\hline Multifocal & 9 & $4(44)$ & $5(56)$ & \\
\hline Tumor diameter $(\mathrm{cm})^{\mathrm{a}}$ & $3.0(0.9-7.5)$ & $2.6(1-5.5)$ & $3.7(0.9-7.5)$ & \\
\hline$<3.0$ & 15 & $9(60)$ & $6(40)$ & 0.199 \\
\hline$\geq 3.0$ & 14 & $5(36)$ & $9(64)$ & \\
\hline \multirow{2}{*}{\multicolumn{5}{|c|}{$\begin{array}{l}\text { Degree of tumor } \\
\text { differentiation }\end{array}$}} \\
\hline & & & & \\
\hline Well & 8 & $7(88)$ & $1(12)$ & 0.005 \\
\hline Moderately & 11 & $6(55)$ & $5(45)$ & \\
\hline Poorly & 10 & $1(10)$ & $9(90)$ & \\
\hline \multicolumn{5}{|c|}{$\begin{array}{l}\text { Tumor invasiveness (vascular invasion } \\
\text { and/or intrahepatic metastasis) }\end{array}$} \\
\hline Negative & 16 & $11(69)$ & $5(31)$ & 0.016 \\
\hline Positive & 13 & $3(23)$ & $10(77)$ & \\
\hline \multicolumn{5}{|c|}{ Fibrous capsular formation/infiltration } \\
\hline Negative & 11 & $3(27)$ & $8(73)$ & 0.216 \\
\hline Positive/negative & 3 & $2(67)$ & $1(33)$ & \\
\hline Positive/positive & 15 & $9(60)$ & $6(40)$ & \\
\hline
\end{tabular}

Data shown are number of cases, and the numbers in parenthesis are percentage of cases unless otherwise indicated. ${ }^{\mathrm{a} D a t a}$ are expressed as

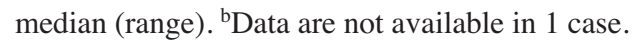

with ECL plus ${ }^{\mathrm{TM}}$ (GE Healthcare) and detected by LAS-1000 (Fujifilm, Tokyo, Japan).

Cell proliferation assay. The human hepatoma cell lines mentioned above were seeded into 96-well tissue culture plates (BD) at 1,000 cells/well in DMEM supplemented with $10 \% \mathrm{v} / \mathrm{v}$ FCS. After 24, 48 and $72 \mathrm{~h}$, the numbers of viable cells were determined by a soluble tetrazolium/formazan assay using Cell Counting Kit-8 (Dojindo Laboratories, Kumamoto, Japan) (15).

Statistical analysis. Statistical analysis was performed using StatView 5.0J software (SAS Institute Inc., Cary, NC, USA).
The Mann-Whitney U test was performed to compare variables of two categories. The Kruskal-Wallis test followed by the Scheffé's post hoc test was performed to compare variables of three or more categories. All statistical analyses were considered to be significant at $\mathrm{p}<0.05$.

\section{Results}

Immunohistochemical staining of a-taxilin in HCCs and noncancerous liver tissues. Diffuse granular staining of $\alpha$-taxilin was observed in the cytoplasm of HCC cells. Fifteen cases were classified as 'strong staining', where all the tumor cells 

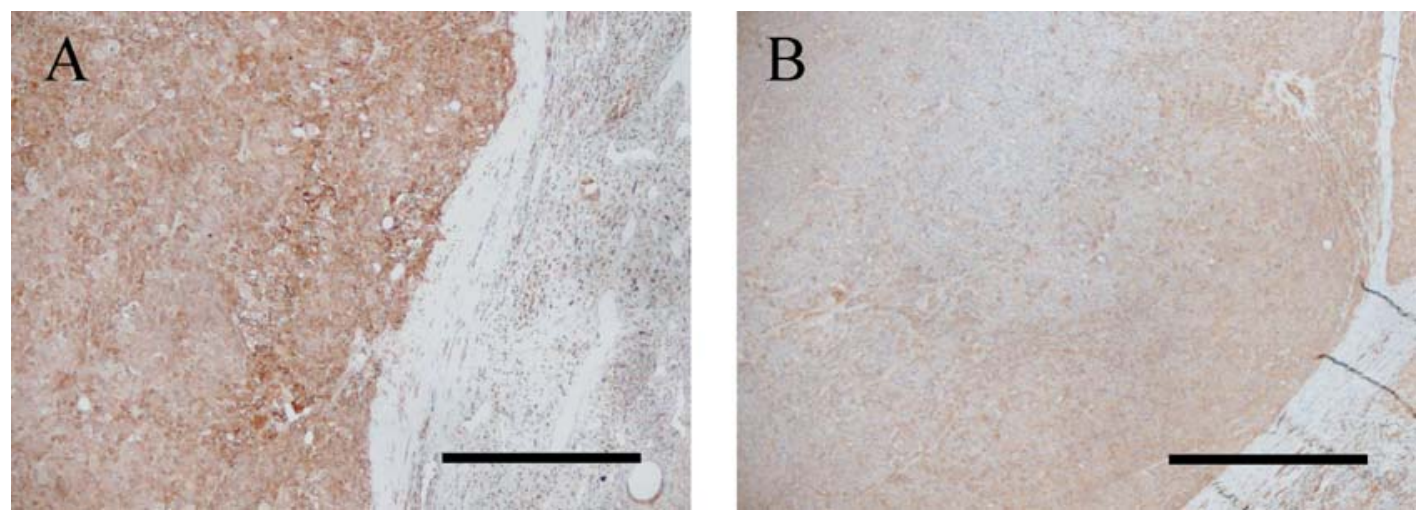

Figure 1. Expression of $\alpha$-taxilin in HCCs. Intensities of staining were classified into 2 categories: (A) strong staining, almost all the cancerous cells were stained; (B) weak staining, no stained cells or few weakly stained cells were observed in cancerous tissues. Bar, $200 \mu \mathrm{m}$.
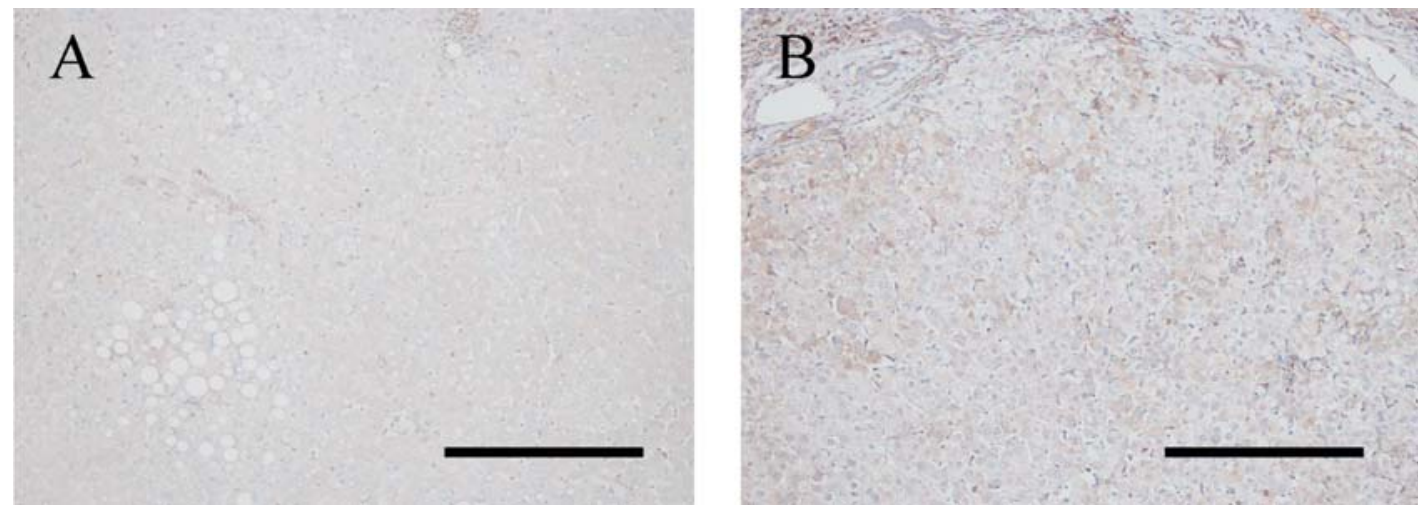

Figure 2. Expression of $\alpha$-taxilin in non-cancerous tissues. Intensities of staining were evaluated as undetectable (A), or weakly detectable (B). Bar, $200 \mu \mathrm{m}$.
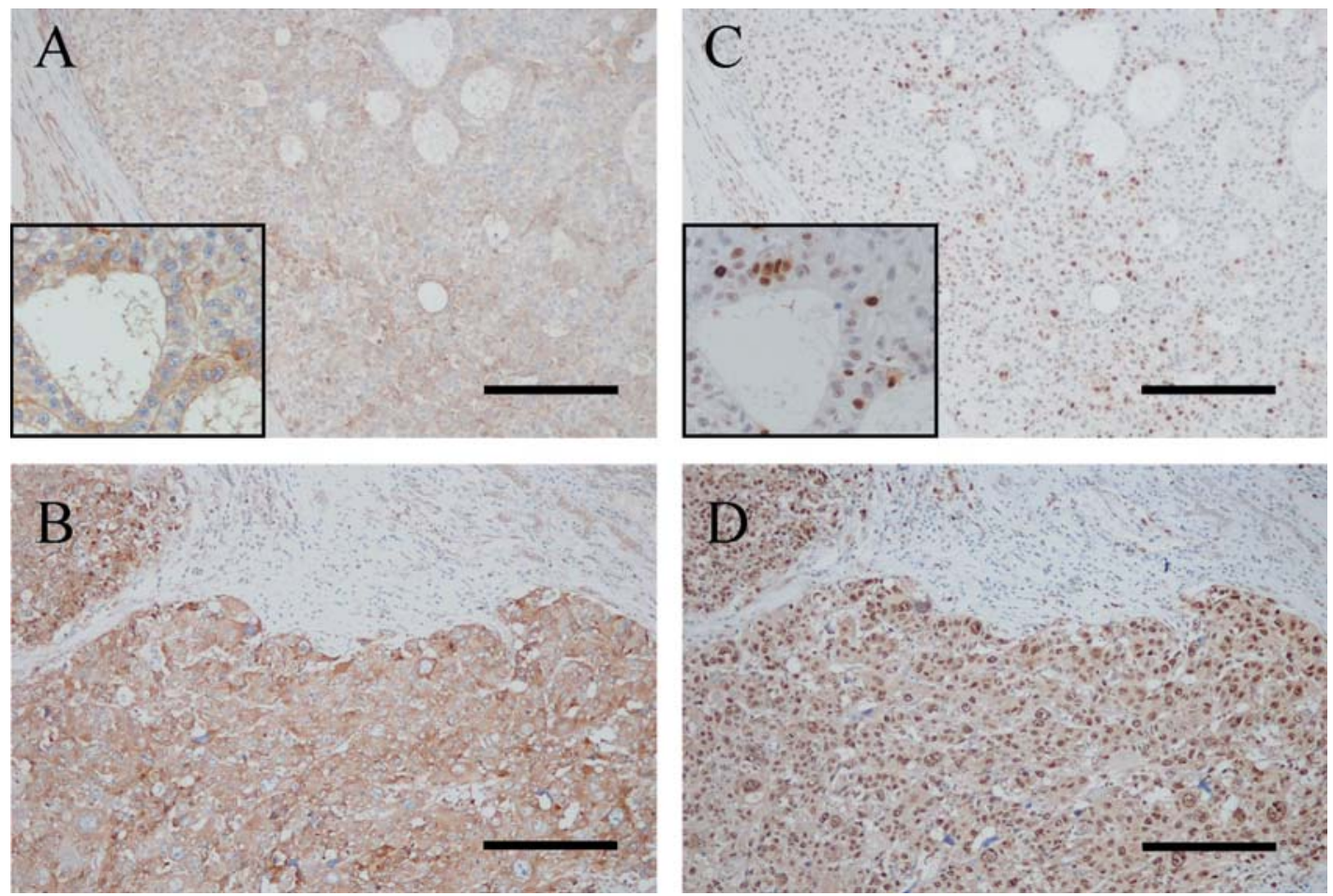

Figure 3. Expression of $\alpha$-taxilin (A and B) and PCNA (C and D) in HCCs. Intensities of $\alpha$-taxilin staining and PCNA-LI were determined in serial sections. Two representative cases are shown. Case 1, weak staining of $\alpha$-taxilin (A) and 7.5\% of PCNA-LI (C). Case 2, strong staining of $\alpha$-taxilin (B) and $94.2 \%$ of PCNA-LI (D). Magnified pictures are shown in boxes (A and C). Bar, $500 \mu \mathrm{m}$. 


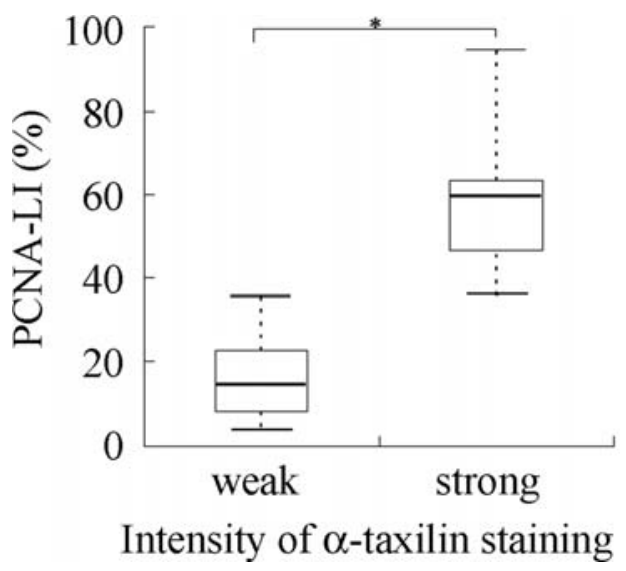

Figure 4. Relationship between $\alpha$-taxilin expression and PCNA-LI. Tukey's box-and whisker plot are shown. Asterisk indicates a significant difference ( $\mathrm{p}<0.0001$ by the Mann-Whitney $\mathrm{U}$ test).

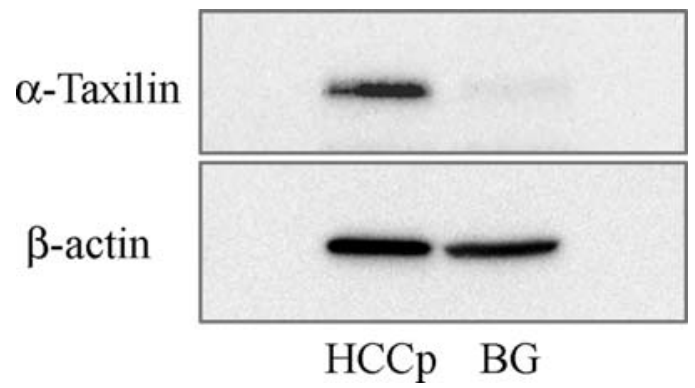

Figure 5. Expression of $\alpha$-taxilin in HCC and surrounding non-cancerous liver tissue, determined by Western blotting. HCCp, cancerous tissue obtained from poorly differentiated $\mathrm{HCC}$; BG, non-cancerous background liver tissue.

were clearly stained with or without strongly stained foci (Fig. 1A). Fourteen cases were classified as 'weak staining', where only a part of tumor cells showed weak staining mainly in periphery of the tumor nodules (Fig. 1B). Relationships between the $\alpha$-taxilin expression and various clinicopathological features were shown in Table I. Significantly higher expression of $\alpha$-taxilin was observed in less differentiated HCCs ( $\mathrm{p}=0.005)$, and more invasive HCCs ( $\mathrm{p}=0.016$ ).

In non-cancerous liver tissues surrounding HCCs, the staining was much weaker compared to HCCs. Eleven cases showed no detectable staining (Fig. 2A), 17 cases showed weak and scattered staining in the cytoplasm of hepatocytes in periportal and periseptal areas (Fig. 2B), and 1 case was not appropriate for evaluation because of the small specimen size of non-cancerous tissue.

Relationship between expressions of $\alpha$-taxilin and PCNA in HCCs. PCNA-LI in HCCs ranged from 3.4 to $94.2 \%$, and the median was $36.0 \%$. $\alpha$-taxilin-positive cells and PCNApositive cells showed very similar distribution in the tumor nodules when comparing serial sections (Fig. 3A and C). The sections of 'strong staining' group of $\alpha$-taxilin showed significantly higher PCNA-LI than the 'weak staining' group (the medians of PCNA-LI were $59.4 \%$ vs. $14.4 \%, \mathrm{p}<0.0001$ ) (Fig. 4).

Detection of $\alpha$-taxilin expression in HCC and non-cancerous tissues by Western blot analysis. Western blot analysis showed a single band consistent with $\alpha$-taxilin (75 kDa) (8) in the tissue of poorly differentiated HCC (Fig. 5), which was strongly stained for $\alpha$-taxilin by immunohistochemistry as shown in Fig. 3B. Much weaker signal was detected by Western blotting in the surrounding non-cancerous tissue.

Relationship between $\alpha$-taxilin expression and cellular proliferation in hepatoma cell lines. All the cell lines in logarithmic-growth phase expressed a single band consistent with $\alpha$-taxilin at various intensities by Western blotting (Fig. 6A and B). The rate of cell growth correlated with the intensity of $\alpha$-taxilin expression (Fig. 6C).
A

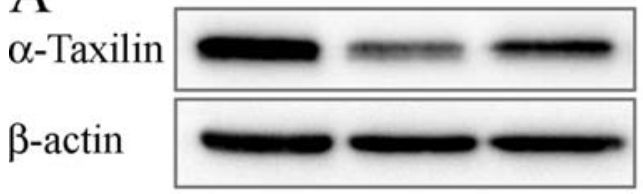

PLC/PRF/5 HuH-6 Hep G2

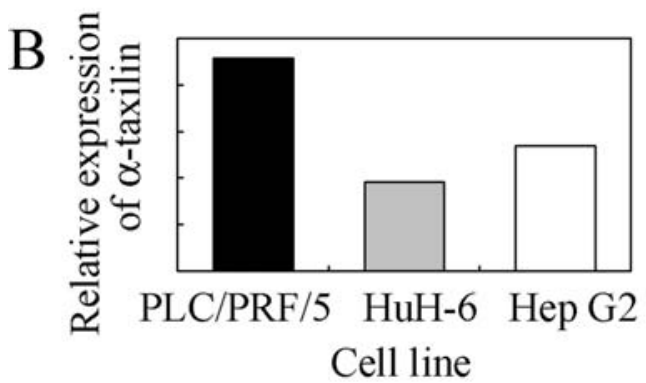

$\mathrm{C}$

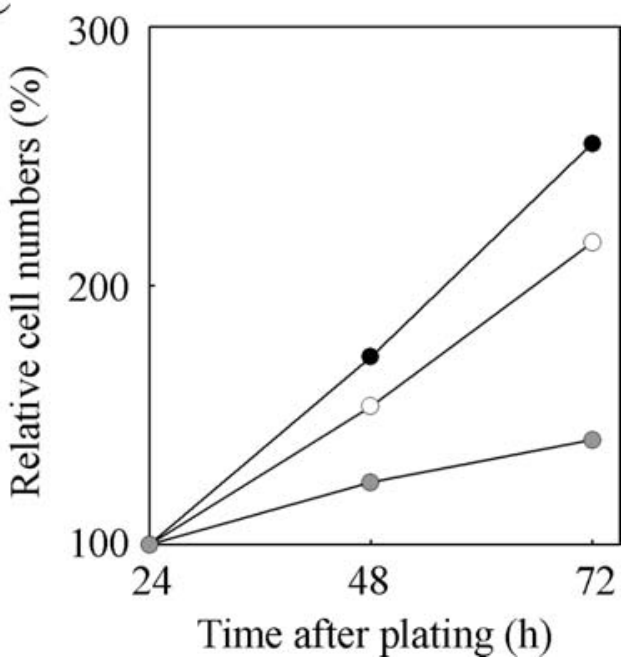

Figure 6. Expression of $\alpha$-taxilin in hepatoma cell lines (A and B) and cell proliferation rate (C). Closed circles, open circles and shaded circles indicate PLC/PRF/5, Hep G2 and HuH-6, respectively. The expression levels of $\alpha$-taxilin in the cell lines were correlated with their growth rates. 


\section{Discussion}

We demonstrated the expression of $\alpha$-taxilin in HCCs, and its expression levels correlated with dedifferentiation, invasiveness and growth activity. We used a rabbit polyclonal anti-human $\alpha$-taxilin antibody. Previous reports have shown a single band in lysates of HeLa and COS-7 cells expressing human recombinant $\alpha$-taxilin by Western blotting using this antibody (12). In the present study, a similar single band consistent with $\alpha$-taxilin molecular weight was detected in HCC specimen and hepatoma cell lines by Western blotting. This antibody detects $\alpha$-taxilin specifically in HCC cells.

We determined proliferative activity of $\mathrm{HCC}$ using PCNALI. PCNA accumulates in the nuclei during S-phase of the cell cycle (16), and is considered as a marker of proliferative activity of various tumors including $\operatorname{HCC}(13,14,17-20)$. Previous studies have reported that PCNA-LI in HCCs ranged from 0.2 to $73.3 \%$ (13), from 1.0 to $89.4 \%$ (17), and from 1.2 to $91.6 \%$ (18). In the present study, PCNA-LI was ranged from 3.9 to $94.2 \%$, which is in line with previous reports. The grade of $\alpha$-taxilin expression in HCCs showed a significant positive correlation with PCNA-LI. In addition, the spatial distribution of $\alpha$-taxilin positive cells was similar with that of PCNA. These findings suggest that expression levels of $\alpha$ taxilin relate to proliferative activity of HCC. This notion is supported by the data of in vitro experiments showing the relationship between the extent of $\alpha$-taxilin and proliferative activity of hepatoma cell lines.

Enhanced $\alpha$-taxilin expression in HCCs was also significantly correlated with less-differentiated histological grade, and more invasive characteristics indicated by positivity for vascular invasion and/or intrahepatic metastasis $(21,22)$. Dedifferentiation of HCCs is usually considered to be associated with higher proliferative activity of the tumor and higher risk of vascular invasion and metastases (2). In addition, in the 'weak staining' cases, $\alpha$-taxilin positive cells tended to distribute in the periphery of tumor nodules. This feature is also reported for several proteins associated with tumor progression and angiogenesis in $\operatorname{HCC}(23,24)$. These findings may suggest that $\alpha$-taxilin expression in $\mathrm{HCC}$ is associated with tumor aggressiveness represented by rapid proliferation and dedifferentiation.

In non-cancerous liver tissues, $\alpha$-taxilin was weakly expressed in small numbers of hepatocytes. These hepatocytes were mainly distributed in periportal and periseptal area of liver lobule, where mitogenic activity of hepatocytes is considered to be high (25). The expression of $\alpha$-taxilin in background liver might relate to the potential of hepatocyte proliferation.

At present, limited data are obtained about taxilin, and the precise function still remains unclear. Taxilin is well preserved between species, and three isoforms $(\alpha-, \beta-, \gamma-)$ were reported in mammals (26). The structure of $\alpha$-taxilin is characterized by a long coiled-coil domain and a leucine zipper motif. Both of them are known to be necessary for a protein-protein interaction or protein dimerization (27), and commonly found in proteins involved in important biological functions such as regulation of gene expression. The long coiled-coil domain is also a characteristic structure of proteins supporting SNARE-mediated membrane fusion (28). The
SNARE proteins are localized on the restricted membrane components, and supposed to have selective function in specific intracellular trafficking steps (7). In vitro binding assay has shown that $\alpha$-taxilin binds with some isoforms of syntaxins, specific members of SNAREs (8), which are predominantly localized on the plasma membrane and involved in post-Golgi vesicle transport. These findings lead one hypothesis that $\alpha$-taxilin is involved in post-Golgi membrane traffic through its association with syntaxins localized on the plasma membrane. In addition, recent studies suggest the involvement of SNAREs and these related molecules in cell proliferation through various steps requiring membrane fusion events, such as nuclear envelope reassembly (29), cytokinesis (29-31), and organelle inheritance. $\alpha$-taxilin might affect tumor growth through membrane fusion event. Furthermore, $\alpha$-taxilin has been reported to have another possible binding partner unrelated to SNAREs. In vitro binding assay has shown that $\alpha$-taxilin binds to nascent polypeptide-associated complex (NAC) (12), a ubiquitous factor of eukaryotic cells. NAC reversibly binds to newly synthesized polypeptide chains, and prevent them from improper folding or unwanted interactions with other proteins (32). This finding suggests the association of $\alpha$-taxilin with translating ribosomes. Diverse functions of $\alpha$-taxilin should be considered to reveal a possible mitogenic effect of $\alpha$-taxilin in tumors.

New aspects have been highlighted about the function of factors associated with membrane traffic, especially SNAREs and these related proteins, in cell growth and organogenesis other than membrane fusion event. While ZW10 binds to syntaxin-18 and play a role in vesicle transport between Golgi and the endoplasmic reticulum (33), it is also known as a mitotic checkpoint protein, controlling attachment of microtubules to kinetochores of chromosomes in Drosophila (34). Syntaxin-2, which is localized on plasma membrane and involved in post-Golgi transport, has also been identified as an extracellular molecule playing a role in morphogenesis of epithelial organs (35) including liver (36). Overexpression of syntaxin- 2 in the mouse mammary gland promotes alveolar hyperplasia and mammary adenocarcinoma (5). Syntaxin-7, which locates on endosome and mediates endosomal/lysosomal fusion events, has been reported to be associated with more aggressive phenotype of malignant melanoma (3). Syntaxin-1 has been associated with more aggressive forms of colorectal carcinomas (37). The relationship between cell proliferation and membrane traffic related proteins would be further investigated in the future.

In conclusion, the expression of the $\alpha$-taxilin protein is enhanced in HCC, and related with increased proliferative activity and dedifferentiation of HCC. In addition, $\alpha$-taxilin can be utilized as a marker of malignant potential of HCC.

\section{References}

1. Pang RW and Poon RT: From molecular biology to targeted therapies for hepatocellular carcinoma: the future is now. Oncology 72 (Suppl. 1): 30-44, 2007

2. Trevisani F, Cantarini MC, Wands JR and Bernardi M: Recent advances in the natural history of hepatocellular carcinoma. Carcinogenesis 29: 1299-1305, 2008.

3. Stromberg S, Agnarsdottir M, Magnusson K, et al: Selective expression of Syntaxin-7 protein in benign melanocytes and malignant melanoma. J Proteome Res 8: 1639-1646, 2009. 
4. Hashimoto S, Onodera Y, Hashimoto A, et al: Requirement for Arf6 in breast cancer invasive activities. Proc Natl Acad Sci USA 101: 6647-6652, 2004

5. Bascom JL, Fata JE, Hirai Y, Sternlicht MD and Bissell MJ: Epimorphin overexpression in the mouse mammary gland promotes alveolar hyperplasia and mammary adenocarcinoma. Cancer Res 65: 8617-8621, 2005.

6. Rothman JE: Mechanisms of intracellular protein transport Nature 372: 55-63, 1994.

7. Chen YA and Scheller RH: SNARE-mediated membrane fusion. Nat Rev Mol Cell Biol 2: 98-106, 2001.

8. Nogami S, Satoh S, Nakano M, et al: Taxilin; a novel syntaxinbinding protein that is involved in $\mathrm{Ca}^{2+}$-dependent exocytosis in neuroendocrine cells. Genes Cells 8: 17-28, 2003

9. Oba-Shinjo SM, Bengtson MH, Winnischofer SM, et al: Identification of novel differentially expressed genes in human astrocytomas by cDNA representational difference analysis. Brain Res Mol Brain Res 140: 25-33, 2005.

10. Sakakibara S, Nakadate K, Tanaka-Nakadate S, et al: Developmental and spatial expression pattern of alpha-taxilin in the rat central nervous system. J Comp Neurol 511: 65-80, 2008.

11. The Liver Cancer Study Group of Japan: The general rules for the clinical and pathological study of primary liver cancer. Kanehara \& Co., Ltd., Tokyo, 2008.

12. Yoshida K, Nogami S, Satoh S, et al: Interaction of the taxilin family with the nascent polypeptide-associated complex that is involved in the transcriptional and translational processes. Genes Cells 10: 465-476, 2005.

13. Kitamoto M, Nakanishi T, Kira S, et al: The assessment of proliferating cell nuclear antigen immunohistochemical staining in small hepatocellular carcinoma and its relationship to histologic characteristics and prognosis. Cancer 72: 1859-1865, 1993.

14. Zeng WJ, Liu GY, Xu J, Zhou XD, Zhang YE and Zhang N: Pathological characteristics, PCNA labeling index and DNA index in prognostic evaluation of patients with moderately differentiated hepatocellular carcinoma. World J Gastroenterol 8: 1040-1044, 2002.

15. Yang L, Yang XC, Yang JK, et al: Cyclosporin A suppresses proliferation of endothelial progenitor cells: involvement of nitric oxide synthase inhibition. Intern Med 47: 1457-1464, 2008.

16. Bravo R, Frank R, Blundell PA and Macdonald-Bravo H: Cyclin/PCNA is the auxiliary protein of DNA polymerase-delta Nature 326: 515-517, 1987.

17. Ng IO, Lai EC, Fan ST, Ng M, Chan AS and So MK: Prognostic significance of proliferating cell nuclear antigen expression in hepatocellular carcinoma. Cancer 73: 2268-2274, 1994

18. Suehiro T, Matsumata T, Itasaka H, Yamamoto K, Kawahara N and Sugimachi K: Clinicopathologic features and prognosis of resected hepatocellular carcinomas of varied sizes with special reference to proliferating cell nuclear antigen. Cancer 76: 399-405, 1995.

19. Taniai M, Tomimatsu M, Okuda H, Saito A and Obata H: Immunohistochemical detection of proliferating cell nuclear antigen in hepatocellular carcinoma: relationship to histological grade. J Gastroenterol Hepatol 8: 420-425, 1993.

20. Yun JP, Miao J, Chen GG, et al: Increased expression of nucleophosmin/B23 in hepatocellular carcinoma and correlation with clinicopathological parameters. Br J Cancer 96: 477-484, 2007.
21. Kosuge T, Makuuchi M, Takayama T, Yamamoto J, Shimada K and Yamasaki S: Long-term results after resection of hepatocellular carcinoma: experience of 480 cases. Hepatogastroenterology 40: 328-332, 1993.

22. Aoki $\mathrm{T}$, Inoue $\mathrm{S}$, Imamura $\mathrm{H}$, et al: EBAG9/RCAS1 expression in hepatocellular carcinoma: correlation with tumour dedifferentiation and proliferation. Eur J Cancer 39: 1552-1561, 2003.

23. Yamamoto S, Tomita Y, Nakamori S, et al: Elevated expression of valosin-containing protein (p97) in hepatocellular carcinoma is correlated with increased incidence of tumor recurrence. J Clin Oncol 21: 447-452, 2003.

24. Yamaguchi R, Yano H, Nakashima O, et al: Expression of vascular endothelial growth factor- $\mathrm{C}$ in human hepatocellular carcinoma. J Gastroenterol Hepatol 21: 152-160, 2006.

25. Harada K, Yasoshima M, Ozaki S, Sanzen T and Nakamura Y: PCR and in situ hybridization studies of telomerase subunits in human non-neoplastic livers. J Pathol 193: 210-217, 2001.

26. Nogami S, Satoh S, Tanaka-Nakadate S, et al: Identification and characterization of taxilin isoforms. Biochem Biophys Res Commun 319: 936-943, 2004

27. Nishizawa M, Kataoka K, Goto N, Fujiwara KT and Kawai S: v-maf, a viral oncogene that encodes a 'leucine zipper' motif. Proc Natl Acad Sci USA 86: 7711-7715, 1989.

28. Cai H, Reinisch K and Ferro-Novick S: Coats, tethers, Rabs, and SNAREs work together to mediate the intracellular destination of a transport vesicle. Dev Cell 12: 671-682, 2007.

29. Jantsch-Plunger V and Glotzer M: Depletion of syntaxins in the early Caenorhabditis elegans embryo reveals a role for membrane fusion events in cytokinesis. Curr Biol 9: 738-745, 1999.

30. Low SH, Li X, Miura M, Kudo N, Quinones B and Weimbs T: Syntaxin 2 and endobrevin are required for the terminal step of cytokinesis in mammalian cells. Dev Cell 4: 753-759, 2003.

31. Prekeris R and Gould GW: Breaking up is hard to do - membrane traffic in cytokinesis. J Cell Sci 121: 1569-1576, 2008.

32. Rospert S, Dubaquie Y and Gautschi M: Nascent-polypeptideassociated complex. Cell Mol Life Sci 59: 1632-1639, 2002.

33. Hirose H, Arasaki K, Dohmae N, et al: Implication of ZW10 in membrane trafficking between the endoplasmic reticulum and Golgi. EMBO J 23: 1267-1278, 2004.

34. Karess R: Rod-Zw10-Zwilch: a key player in the spindle checkpoint. Trends Cell Biol 15: 386-392, 2005.

35. Chen CS, Nelson CM, Khauv D, et al: Homology with vesicle fusion mediator syntaxin-1a predicts determinants of epimorphin/syntaxin-2 function in mammary epithelial morphogenesis. J Biol Chem 284: 6877-6884, 2009.

36. Miura K, Yoshino R, Hirai Y, et al: Epimorphin, a morphogenic protein, induces proteases in rodent hepatocytes through NFkappaB. J Hepatol 47: 834-843, 2007.

37. Grabowski P, Schonfelder J, Ahnert-Hilger G, et al: Expression of neuroendocrine markers: a signature of human undifferentiated carcinoma of the colon and rectum. Virchows Arch 441: 256-263, 2002. 